\title{
Biometric Gait Recognition with Carrying and Clothing Variants
}

\author{
Shamsher Singh and K.K. Biswas \\ Dept. of CSE, IIT Delhi-110016, India \\ sskarki@gmail.com, kkb@cse.iitd.ac.in
}

\begin{abstract}
Compact spatio temporal representation of human gait in form of gait enery image (GEI) has attracted lot of attention in recent years for biometric gait recognition. Researchers have reported very high recognition rates for normal walk sequences. However, the rates come down when the subjects are wearing a jacket or coat, or are carrying a bag. This paper shows that the performance for the variant situations can be improved upon considerably by constructing the GEI with sway alignment instead of upper body alignment, and selecting just the required number of rows from the bottom of the silhouette as inputs for an unsupervised feature selection approach. The improvement in recognition rates are established by comparing performances with existing results on a large gait database.
\end{abstract}

\section{Introduction}

Biometric gait recognition refers to identifying a person from his normal walking style (gait). This is a marker less unobtrusive biometric which can be done at a distance and does not need user interaction or cooperation. [1] 2, 3] 4] [5]

Recently there has been a lot of interest in using gait energy as a feature for gait based recognition 6] [7] 8 . The silhouettes of a person are extracted from each frame of a walking cycle and superimposed on the first frame after aligning the upper body of the person. This single image is referred to as Gait Energy Image (GEI). This represents the walking dynamics of the person in a compact manner. It has been shown that this representation is less sensitive to noise and recognition rates are very promising for normal walk sequences [6] [7] []. However the performance of the recognition system goes down if the person is wearing a coat or is carrying a shoulder bag. This paper shows that by aligning the silhouettes in a slightly different manner and with a proper selection of energy points from the GEI, the gait recognition performance can be improved for clothing and carrying bag variants of walking. The performance has been measured with the same data set as used in Bashir [8].

\section{Generating the GEI}

We assume that the silhouettes of a person have already been extracted from the walk sequence video clips. Many data bases which are available online, provide

S. Chaudhury et al. (Eds.): PReMI 2009, LNCS 5909, pp. 446-451, 2009.

(C) Springer-Verlag Berlin Heidelberg 2009 
such silhouettes. We have chosen the CASIA database [9] for our study. We define a walk cycle to consist of frames between two identical key frames consisting of legs together stances of a person. Using the white pixel coverage of lower portion of the body one can determine these key frames. We then normalize the silhouette height to a window of height 100 pixels.

The conventional way of projecting the silhouettes is to align the head and upper torso of the subject in each frame with the corresponding torso of the first frame. In this approach apart from dynamic hand motion information the upper body part appears static in the final GEI. However, as a person walks, his whole body sways forward and backward periodically and this information is lost in the simple alignment technique. This alignment also influences the shape of the the dynamic area of the lower body part as well, which is critical in defining a persons gait characteristics.

We propose a method of alignment that takes into consideration the whole body motion. For each frame we use the maximum and minimum horizontal points of the silhouette to compute the horizontal centroid and use this to align the silhouettes. These points do not necessarily belong to upper body part and hence depicts true horizontal centroid. We refer to this as "sway alignment" in our work.

\section{Selection of Feature Points}

To reduce the dimensionality of feature points, PCA is employed both for unsupervised or supervised selection approaches [7] []. Supervised feature selection is tried on the bottom half of the GEI in $[8]$. It is obvious that more useful gait information is contained in the lower part of the body, particularly the legs. Also it should be noted that this part is usually unaffected, if the person wears a coat or a jacket or is carrying a bag on his shoulders. Thus we concentrate on the dynamic lower part by selecting a suitable area from the bottom of the silhouette, The motivation behind this is to use the pure gait information without the influence of shape. Also, it drastically reduces the number of feature points needed for training.

To get an approximate position of the knee, we first locate the lowest position of the palm using the golden ratio (1.618) of the human body[10]. As the silhouette height is 100 pixels in our case, we get palm position at row 62 from the top of the head. The knee would thus be located within 38 rows from the bottom of the silhouette. Our desired dynamic area now consists of silhouette rows between the ground level and a chosen limiting row. We name the limiting row as $R_{\text {lim }}$ (which is less than 38 ). By carrying out exhaustive experimentation we found that $R_{\text {lim }}$ can be set comfortably between 25 and 30 . We keep the $R_{\text {lim }}$ fixed for all the gallery and probe sequences of all the persons.

\section{Similarity Analysis}

\subsection{Dataset}

We are using the CASIA dataset created by the center for biometric and security research 9. This database involves 124 subjects and was created with uniform 
background and controlled lighting condition and with different viewing angles. The Database consists of three variants which are carrying bag, different clothing and view angle. For first two variants 2 walk per person are available while 11 cameras at different angles are used for each walk. The starting stance of the walks is not fixed.

Our approach is aimed for single camera based systems and applicable in scenarios where restrictive entry is allowed. In GEI based approaches good results are reported when view angle is $90^{\circ}$. We have used the silhouettes of $90^{\circ}$ view angle from the dataset. The data is partitioned in three sets.

set A: Six normal walks for the same person

set B: Two walks by the same person but now carrying a bag

set $\mathrm{C}$ : Two walks by the same person with clothing change

To do the similarity analysis the whole data is divided into probe and gallery. The gallery set $(\mathrm{Ag})$ is made of first four normal walks for each person. We chose this data set to compare our results with existing methods where the same very walks are used as the gallery set 8 . As our interest is to show that recognition can be carried out even when a person is carrying a bag, the Probe data (for recognizing a person) consists of $3 \operatorname{sets}(\mathrm{Ap}, \mathrm{B}, \mathrm{C})$. Ap happens to be the last two walks of 6 normal walk data of set A. For both the probe and gallery sequence, we have generated two GEIs per walk, belonging to alternate gait cycles.

\subsection{Analysis}

As mentioned earlier, we are only using the feature points from dynamic area of GEI,bounded by $R_{\text {lim }}$. Similarity between probe and gallery walks is estimated by calculating the normalized sum of absolute differences between the Gray values of two GEI as shown in equation 11. Nearest neighbor classifier is then used for recognition. Figure 1 depicts the process of similarity analysis between the gallery sequence of subject ${ }_{i}$ and the $k^{\text {th }}$ probe sequence subject $_{j}$. As mentioned earlier for each subject four walk sequence of gallery and two walk sequence of probe are used where each walk sequence consists of two GEIs.

$$
\begin{array}{r}
\text { Diff } f_{i}=\operatorname{Abs}\left(g_{i}(p)-g_{i}(g)\right) \\
\text { Diff }=\Sigma_{i}^{n} \text { Diff } f_{i} / n
\end{array}
$$

$g_{i}(p)$ : Gray value at location i for probe $\mathrm{p}$

$g_{i}(g)$ : Gray value at location i for gallery g

Diff : Normalized difference between probe(p) and gallery $(\mathrm{g})$

$\mathrm{n}$ : Number of points used, depends on $R_{\text {lim }}$

For each of the two probe sequences we calculate average difference with the gallery as shown in equation 2

$$
\operatorname{AvgDiff}\left(p r_{k}\right)=M I N 1+M I N 2 / 2
$$

$\mathrm{k}: 1,2$ denote two probe sequence

MIN1, MIN2: Least 2 values among Min(a1,b1), Min(a2,b2), Min(a3,b3) and $\operatorname{Min}(\mathrm{a} 4, \mathrm{~b} 4)$ 


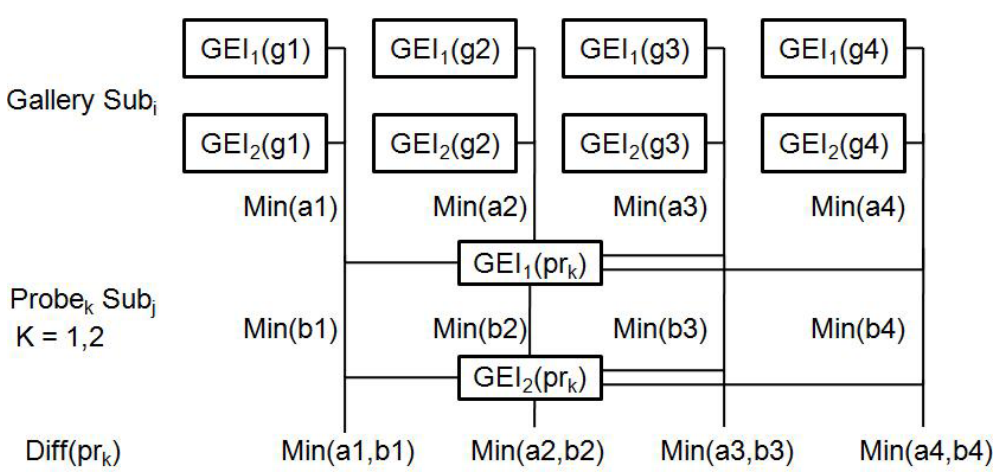

Fig. 1. Similarity analysis

Final similarity between Gallery $S u b_{i}$ and Probe $S u b_{j}$ is estimated by calculating the minimum average difference among the two probe sequences as show in equation 3 .

$$
\operatorname{sim}\left(S u b_{i}, S u b_{j}\right)=\min \left(\operatorname{Avg} \operatorname{Diff}\left(\operatorname{pr}_{k}\right)\right)
$$

\section{Performance Evaluation}

We have evaluated the performances of performed the gait analysis using upper body alignment and using our "sway alignment". For the CASIA dataset database we have calculated recognition rates for various rank values. Rank N recognition indicates that the person in the probe set is present among the top $\mathrm{N}$ similarity scores from the gallery.

We have performed the gait analysis using both the upper body alignment as well as sway alignment. We have calculated rank 1 , rank 2 and rank 5 identification rates for each of the variant. Table 1 presents the results for $R_{\text {lim }}=30$ and table 2 shows the results for $R_{\text {lim }}=25$. It is observed that in each case the sway alignment performs better in case of normal walk(set Ap) and clothing(set C) variant but upper body alignment performs better in case of carrying bag(set B) condition.

Comparison with existing results. We have compared the results of our experiments with CAS(Chinese Academy of Sciences): GEI shape match approach [6], ], UCR(Univ. of California Riverside): PCA and MDA base feature learning method [7] and (Queen Marys, Univ of London): Feature selection based approach 8 . We have used the result of sway alignment for normal walk and clothing variant while upper body alignment based results have been presented for carrying bag variant. We have used Rlim as 30 for our results.

Both the CAS and UCR methods use the whole of the GEI for analysis. UCR employs PCA and MDA for feature learning. Table 3 presents the comparison 
Table 1. Results: Static feature $\operatorname{selection}\left(R_{\text {lim }}=30\right)$

\begin{tabular}{|l||l||l|l|l||}
\hline Method & Identification rate & Set Ap & Set B & Set C \\
\hline Upper body alignment & Rank 1 & $\mathbf{8 8 . 5 2}$ & $\mathbf{7 4 . 5 9}$ & $\mathbf{5 8 . 1 9}$ \\
\cline { 2 - 5 } & Rank 2 & 92.80 & 79.50 & 69.66 \\
\cline { 2 - 5 } & Rank 5 & 96.72 & 82.79 & 73.77 \\
\hline \hline \multirow{3}{*}{ Sway alignment } & Rank 1 & $\mathbf{9 3 . 4 4}$ & $\mathbf{4 7 . 5 4}$ & $\mathbf{7 7 . 0 4}$ \\
\cline { 2 - 5 } & Rank 2 & 95.9 & 59.83 & 86.06 \\
\cline { 2 - 5 } & Rank & 96.72 & 68.85 & 87.70 \\
\hline
\end{tabular}

Table 2. Results: Static feature $\operatorname{selection}\left(R_{\text {lim }}=25\right)$

\begin{tabular}{|l||l||l|l|l||}
\hline Method & Identification rate & Set Ap & Set B & Set C \\
\hline \multirow{3}{*}{ Upper body alignment } & Rank 1 & $\mathbf{8 5 . 2 5}$ & $\mathbf{7 7 . 0 5}$ & $\mathbf{5 4 . 9 2}$ \\
\cline { 2 - 5 } & Rank 2 & 90.16 & 81.15 & 67.21 \\
\cline { 2 - 5 } & Rank 5 & 94.26 & 89.34 & 78.69 \\
\hline \hline \multirow{3}{*}{ Sway alignment } & Rank 1 & $\mathbf{9 0 . 1 6}$ & $\mathbf{5 0 . 8 1}$ & $\mathbf{7 6 . 2 3}$ \\
\cline { 2 - 5 } & Rank 2 & 94.26 & 59.83 & 82.79 \\
\cline { 2 - 5 } & Rank 5 & 95.9 & 70.49 & 86.88 \\
\hline
\end{tabular}

Table 3. Comparison with existing GEI based approaches

\begin{tabular}{|l||l|l|l|l||}
\hline \multicolumn{1}{|l||}{} & \multicolumn{3}{|l||}{ Existing Method } & Proposed Method \\
\cline { 2 - 4 } & CAS & UCR & QMUL(unsup) & \\
\hline \hline Set Ap & 97.6 & 99.4 & 99.4 & $\mathbf{9 3 . 4 4}$ \\
\hline Set B & 32.7 & 60.2 & 79.9 & $\mathbf{7 4 . 5 8}$ \\
\hline Set C & 52.0 & 22.0 & 31.3 & $\mathbf{7 7 . 0 4}$ \\
\hline
\end{tabular}

with these techniques. In case of normal walk(set Ap) our results are comparable to these approaches. When we analyze the difficult cases of carrying bag(set B) and clothing(set C) variant our approach shows significant improvement. This comparison supports our contention that features from lower leg part are sufficient for recognition and are less sensitive to variations from normal walk sequences. We have also shown the comparison with QMUL:Feature selection based approach of Bashir et al. [8]. Table 3 shows that while the performance of our approach is comparable to the performance of their unsupervised approach for the normal walk and carrying bags, there is a significant improvement for the variants of clothing. Our unsupervised approach also takes less time in comparison.

\section{Conclusion}

In this paper we have shown that the dynamic area of GEI near the foot can be effectively used to improve the recognition rate. This area is comparatively less 
sensitive to clothing and other changes. We have proposed construction of the GEI using our proposed "sway alignment" instead of the conventional "upper body alignment", and shown that selection of the area near the foot considerably improves the results. We have shown considerable improvement in recognition rates for variants over other existing GEI based approaches for a large gait dataset.

\section{References}

1. Liu, Z., Sarkar, S.: Improved gait recognition by gait dynamics normalization. IEEE Trans. Pattern Anal. Mach. Intell. 28(6), 863-876 (2006)

2. Ioannidis, D., Tzovaras, D., Damousis, I.G., Argyropoulos, S., Moustakas, K.: Gait recognition using compact feature extraction transforms and depth information. IEEE Transactions on Information Forensics and Security 2(3-2), 623-630 (2007)

3. Ekinci, M.: Gait recognition using multiple projections. In: FGR 2006: Proceedings of the 7th International Conference on Automatic Face and Gesture Recognition, Washington, DC, USA, pp. 517-522. IEEE Computer Society, Los Alamitos (2006)

4. Nandini, C., Kumar, C.R.: Comprehensive framework to gait recognition. Int. J. Biometrics 1(1), 129-137 (2008)

5. Sundaresan, A., Chowdhury, A.R., Chellappa, R.: A hidden markov model based framework for recognition of humans. In: Proc. ICIP, pp. 93-96 (2003)

6. Yu, S., Tan, D., Tan, T.: A framework for evaluating the effect of view angle, clothing and carrying condition on gait recognition. In: ICPR 2006: Proceedings of the 18th International Conference on Pattern Recognition, Washington, DC, USA, pp. 441-444. IEEE Computer Society, Los Alamitos (2006)

7. Han, J., Bhanu, B.: Individual recognition using gait energy image. IEEE Trans. Pattern Anal. Mach. Intell. 28(2), 316-322 (2006)

8. Bashir, K., Xiang, T., Gong, S.: Feature selection on gait energy image for human identification. In: IEEE International Conference on Acoustics, Speech and Signal Processing, XXX (April 2008)

9. Center for Biometrics and Security Research, CASIA, http://www.cbsr.ia.ac.cn/

10. http://goldennumber.net/body.htm 